\title{
A IMPORTÂNCIA DA TÉCNICA CIRÚRGICA NA MAMOPLASTIA
}

\author{
BIBLIOGRAPHIC REVIEW: BIBLIOGRAPHIC REVIEW: \\ THE IMPORTANCE OF SURGICAL TECHNIQUE IN MAMOPLASTY
}

\author{
Marcela Lordello Alonso ${ }^{1 *}$, Maria Fernanda Vilar Ribeiro', Pedro Murad de Azevedo', Pillar Souza Viana Cancio', \\ Rodrigo Araújo Guimarães². \\ 1 Acadêmicos de Medicina da Faculdade de Medicina de Campos - FMC \\ 2 Médico Esp. em Cirurgia Plástica
}

*Autor de correspondência: Marcela Lordello Alonso. Endereço: Rua pereira nunes, 89. Telefone: (22) 998248487. E-mail:marcelalordello@yahoo.com.br

\section{RESUMO}

Com o avanço e desenvolvimento de novas e diferentes técnicas cirúrgicas, os cirurgiões plásticos, atualmente, conseguem ter uma ampla categoria de vias de acesso cirúrgica, planos e formatos de prótese para realizar as cirurgias. $\mathrm{Na}$ hora da escolha de qual método cirúrgico irá ser utilizado em uma mamoplastia, tem que ser levado em conta muitos fatores que dependem da individualidade e complexidade de cada caso e paciente.

Palavras-chave: Mamoplastia. Planos cirúrgicos. Complicações pós mamoplastia. Cicatrização da mamoplastia.

\begin{abstract}
With the advancement and development of new and different surgical techniques, plastic surgeons, today, are able to have a wide category of surgical access routes, plans and prosthesis formats to perform the surgeries. When choosing which surgical method to use in a mammoplasty, many factors have to be taken into account depending on the individuality and complexity of each case and patient.
\end{abstract}

Keywords: Mammoplasty. Surgical plans. Complications after mammoplasty. Healing of mammoplasty. 


\section{INTRODUÇÃO}

A gratidão das pacientes que realizaram cirurgia plástica estética, em particular, nas mamas, está sempre voltada a qualidade de vida e resultados, com baixos índices de complicações e de reintervenções (ABOUDIB; ROXO, 2011). O intuito é buscar uma técnica cirúrgica associada a um menor número de cicatrizes e manutenções da sensibilidade cutânea e funcional.

Diversos fatores podem afetar resultados, como exemplo: plano para localização do implante, orientação adequada ao paciente, seleção dos pacientes, melhor plano de incisão, técnicas cirúrgicas avançadas, cuidados pós-operatórios, tamanho do tórax, simetrias e características físicas do paciente, são um dos fatores mais importantes para uma boa recuperação. (ABOUDIB; ROXO, 2011). A satisfação do paciente no pós-cirúrgico é alta, apesar de ainda existirem índices de correções de assimetria, contratura capsular, cicatrizes hipertróficas ou outras complicações.

Em relação às técnicas cirúrgicas, as mais populares vias de acesso são: as axilares, inframamárias e periareolares, associadas a inclusão de próteses mamárias. Já os planos utilizados são: o subpeitoral (SP) ou duplo plano, subglandular (SG) e o subfascial (SF). (SHEN et al., 2019). Os formatos de próteses mamárias são: a redonda e a anatômica.

\section{OBJETIVOS}

Esta revisão bibliográfica tem como objetivo a análise e comparação de dados da literatura a fim de evidenciar a relação da escolha de uma técnica cirúrgica melhor e sua interferência nos resultados e complicações das cirurgias de mamoplastia.

\section{MÉTODOS}

Este estudo constitui-se de uma revisão da literatura, realizada entre julho e agosto de 2020, onde foi realizado pesquisas a artigos e publicações disponíveis em revistas científicas e na internet.

A pesquisa dos materiais específicos foi baseada no tema inicial proposto, com a utilização de palavraschave como "mamoplastia", "planos cirúrgicos", "complicações pós mamoplastia" e "cicatrização da mamoplastia".

Em seguida, as publicações selecionadas foram estudas e comparadas buscando encontrar informações que demonstrassem qual técnica foi a menos relacionada a complicações e insatisfações.

\section{RESULTADOS}

Após a seleção e análise dos dados, foi obtido o total de 751 mamas para a realização do estudo das técnicas cirúrgicas. Em seguida, 309 pacientes foram analisadas, onde 33 dessas apresentaram diversos tipos de complicações.

Quando analisada a necessidade de uma segunda abordagem cirúrgica nas pacientes submetidas a mamoplastia prévia, observamos que os motivos podem ser estéticos ou por complicações cirúrgicas (MAXIMILIANO et al., 2017).

A incidência das reintervenções e sua relação com a escolha da técnica cirúrgica esta evidenciada na tabela 1.

Tabela 1: análise de técnicas cirúrgicas de mamoplastia e o IR

\begin{tabular}{cc}
\hline Técnica cirúrgica & IR \\
\hline Mastopexia associada à implante & $5,99 \%$ \\
Mamoplastia redutora periareolar & $3,19 \%$ \\
Mamoplastia de aumento via axilar & $0,53 \%$ \\
Mamoplastia de aumento via transareolopapilar & $3,19 \%$ \\
Mamoplastia de aumento subglandular & $5,99 \%$ \\
Mamoplastia de aumento “dual plane” & $4,2 \%$ \\
\hline IR: Índice de Reintervenção
\end{tabular}

Maximiliano et al. fez um comparativo entre mamoplastia de aumento isolada ou associada à mastopexia. Em relação a necessidade de reintervenção, os resultados obtidos, mesmo sem diferença estatística, mostram que as mulheres que fizeram mastopexia necessitaram de mais reabordagens. Dentre as noves reintervenções citadas no estudo, observaram causas como hematoma ( $1 \%)$, seroma (4\%), mau posicionamento da prótese (2\%) e contratura capsular (3\%). A complicação mais prevalente foi a dor e foi mais prevalente no grupo de implante isolado.

No grupo da mamoplastia de aumento isolado foi mais indicado o plano duplo (45\%) e o subglandular (44\%), já na mastopexia associada ao implante o plano subglandular foi indicado em $71 \%$ das abordagens. No comparativo geral, $56 \%$ das operações foram feitas no plano subglandular e 14\% necessitaram de reintervenção; a técnica de duplo plano foi utilizada em $32 \%$ das cirurgias com $19 \%$ de reintervenção; e o plano subfascial foi escolhido em $12 \%$ dos casos e nenhum passou por reoperação 
(MAXIMILIANO et al., 2017).

Shen et al. faz um estudo associando as técnicas em planos subglandular (SG), subpeitoral (SP) e subfascial (SF) com a incidência de contratura capsular, hematoma, seroma, infecção, reoperação, ondulação, dormência do mamilo, má colocação e ruptura. A contratura capsular foi a principal complicação após a mamoplastia, se formando em 3 meses a 1 ano após a operação (SHEN et al., 2019).

A colocação em SG aumentou o risco de contraturas capsulares, hematomas e seromas em comparação aos outros dois. Já os planos SP e SF se demonstraram ser seguros e eficazes para controlar complicações pós-operatórias e alcançar maior satisfação pós-operatória das pacientes, porém os dos benefícios a longo prazo da técnica SF requerem pesquisa adicional (SHEN et al., 2019).

Em Santo et al., dos 231 pacientes abordados por via axilar em plano subfascial, foram relatados apenas 4 casos de contratura capsular, 2 hematomas, 2 casos de estrias periareolares, 2 casos de seroma e 2 casos de aderência na cicatriz axilar que não precisam de intervenção cirúrgica. Não houveram relatos de migração de implante, infecção, deiscência ou necrose e a perda de sensibilidade aréolo-mamilar foi transitória, quando presente. A abordagem em plano SF permite um acesso com boa visualização para o implante mamário, adequada hemostasia e confere segurança de menor chance de deslocamento do implante, mesmo para os de grande volume (SANTO et al., 2010).

Em teoria, a ocorrência de hematomas e seromas deveriam ser menores no plano SG por se tratar de um plano avascular, sendo facilmente dissecado e com menos lesão tecidual. Entretanto, por razões não explicadas, os resultados obtidos pelo estudo demostraram uma maior incidência, com significância, de hematoma e seroma no plano SG e uma menor no plano SF. (SHEN et al., 2019).

Com a utilização da técnica via transareolopapilar, as principais causas para a necessidade de uma reabordagem foram desenvolvimento de nódulo mamário unilateral $(1,9 \%)$, infecção $(1,9 \%)$, contratura capsular unilateral $(1,9 \%)$ e insatisfação com a forma das mamas $(7,6 \%)$, volume $(7,6 \%)$ e cicatriz $(1,9 \%)$.Nenhuma paciente desenvolveu complicação grave, porém houve relato de maceração da pele no ato da passagem do implante, extrusão de pontos, hipocromia da cicatriz, cicatriz hipertrófica, retração cicatricial e aréola bífida (VERBICARIO et al., 2011).

$O$ acesso via axilar proporciona qualidade e melhor aspecto por deixar as mamas sem cicatrizes, porém a via mais utilizada pelos cirurgiões brasileiros é a incisão inframamária que permite o acesso direto ao plano de colocação e sua cicatriz é curta posicionada no sulco inframamário (SANTO et al., 2010)

$\mathrm{Na}$ associação entre o formato do implante e necessidade de reintervenção não houve significância estatística, $86 \%$ escolheram a prótese redonda e $14 \%$ escolheram o implante anatômico, $16 \%$ com a prótese redonda passou por uma segunda intervenção e nenhum do grupo da prótese anatômica precisou (MAXIMILIANO et al., 2017).

Dessa forma, na tabela 2 foram descritas as complicações e analisado suas taxas de incidência respectivamente.

\begin{tabular}{|c|c|}
\hline Complicações & TI \\
\hline Maceração da pele & 8 \\
\hline Extrusão dos pontos & 3 \\
\hline Seroma & 4 \\
\hline Dor & 2 \\
\hline Cisto cicatricial & 1 \\
\hline Contratura capsular & 4 \\
\hline Hematoma & 3 \\
\hline Aderência axilar & 2 \\
\hline Estrias & 2 \\
\hline Diminuição da sensibilidade & 2 \\
\hline Ressecção insuficiente & 2 \\
\hline Total & 33 \\
\hline
\end{tabular}

\section{CONCLUSÃO}

O conhecimento de todas as vias de acesso, planos e tipos de prótese associado a uma boa anamnese, avalição da anatomia e expectativa do paciente, não mostra a escolha da melhor técnica cirúrgica para diminuir complicações e reoperações. 


\section{REFERÊNCIAS}

MAXIMILIANO, João et al. Mamoplastia de aumento: correlação entre o planejamento cirúrgico e as taxas de complicações pós-operatórias. Rev. Bras. Cir. Plást., Porto Alegre, p. 332-339, 9 jul. 2017.

ABOUDIB, José Horácio; ROXO, Ana Claudia Weck. Avaliação dos resultados tardios de mamoplastia redutora pela técnica periareolar. Rev. Bras. Cir. Plást., São Paulo, v. 26, ed. 1, Jan./Mar. 2011. DOI https://doi.org/10.1590/S198351752011000100016. Disponível em: https://www.scielo.br/scielo.php?pid=S1983$51752011000100016 \&$ script=sci_arttext\&tlng=pt. Acesso em: 23 jul. 2020.

SHEN, Zeren et al. A comparative assessment of three planes of implant placement in breast augmentation: A Bayesian analysis. Journal of Plastic, Reconstructive \& Aesthetic Surgery, China, p. 1-10, 9 set. 2019.

VERBICARIO, João Paulo Mendes et al . Incisão transareolopapilar para mamoplastia de aumento: experiência dos últimos 10 anos do Instituto Ivo Pitanguy. Rev. Bras. Cir. Plást., São Paulo, v. 26, n. 4, p. 670-674, Dec. 2011. Available from <http://www.scielo.br/scielo.php?script=sci_arttext\&pid=S1983$51752011000400023 \&$ Ing=en\&nrm=iso>. access on 02 Aug. 2020. http://dx.doi.org/10.1590/S198351752011000400023.

Daar DA, Abdou SA, Rosario L., Rifkin WJ, Santos PJ, Wirth GA Existe local de incisão preferido para mastectomia poupadora de mamilo? Uma revisão sistemática e meta-análise. Plast Reconstr Surg. 2019; 143 (5): 906e - 919e. PubMed

ESPIRITO SANTO, Paulo Rogério Quieregatto do. Mastoplastia de aumento via axilar: avaliação da técnica subfascial. Rev. Bras. Cir. Plást. (Impr.), São Paulo, v. 25, n. 4, p. 663-667, Dec. 2010 . Available from <http://www.scielo.br/scielo.php?script=sci_arttext\&pid=S1983-51752010000400018\&lng=en\&nrm=iso>. access on 02 Aug. 2020. http://dx.doi.org/10.1590/S1983-51752010000400018

GRAF, Ruth et al . Incisão periareolar em zigue-zague modificada: técnica alternativa para mastoplastia de aumento. Rev. Bras. Cir. Plást., São Paulo, v. 28, n. 2, p. 297-300, June 2013 . Available from <http://www.scielo.br/scielo.php?script=sci_arttext\&pid=S1983-51752013000200022\&lng=en\&nrm=iso>. access on 02 Aug. 2020. https://doi.org/10.1590/S1983-51752013000200022 